\title{
DIARRHOEA IN ADULTS-SOME CAUSES AND THEIR TREATMENT
}

\author{
By R. HALE-WHITE, M.C., M.A., M.D.
}

The cause of diarrhoea is seldom difficult to find; often it is obvious from the history, if it is not the patient and his stools must be examined further. It would be tedious to go through the various methods of examination and the many obvious steps leading to a diagnosis, and it would be even more tedious to give a list of causes of diarrhoea with the differential diagnosis, as this could not be done without endless repetition. In this article five different topics are examined briefly, each one about a single or a group of disorders in which diarrhoea is a leading symptom. The treatment of the various conditions is given as they occur and at the end there is a note on the symptomatic treatment of diarrhoea. In the course of these discussions most of the important causes of diarrhoea in this country are mentioned, but none of those that only occur abroad.

\section{DIARRHOEA DUE TO FOOD}

Children often give themselves diarrhoea through ignorance, naughtiness or bravado, when they eat such things as toadstools, various berries, unripe, overripe, or too much fruit. In adults diarrhoea is common enough through excessive or foolish drinking, but as a result of gluttony alone it is rare. However, food which has gone bad can cause anything from the most trivial digestive upset to death. It is nearly always wrong to call this ptomaine poisoning. Ptomaines are found in the decomposition of food, but even such food does not usually cause any symptoms unless it is contaminated by pathogenic bacteria, such as Salmonella. Dysentery and typhoid are of course caused in this way, but they are not included in what is commonly termed "food poisoning." Heat and flies predispose to such contamination, which occurs usually in meat, fish and milk. Of these, meat and fish are ordinarily cooked before being eaten and in this way the bacteria and their toxins are probably destroyed. More perfect storage, more rapid transport of food and more hygienic methods used by the consumer are all tending to reduce the incidence of food poisoning, but cases are still fairly numerous. When they do occur it is sometimes difficult to know where to put the blame. The ingestion of anything out of a tin used, if possible, to be fastened on as the certain cause. Years ago this may sometimes have been justified. For many more years the prejudice remained, but now it is almost certain that if poisoning is caused by tinned food it is not the fault of the manufacturers, but of the consumer for not eating it quickly enough after the tin is opened.

Often the diagnosis is obvious; often all those who have shared a meal obligingly share the subsequent symptoms. But if one man has symptoms and the rest have not, that in itself does not disprove poisoning, for he may be more susceptible, or, perhaps not all the food in the offending dish was bad. The symptoms usually begin in under six hours, seldom in more than twelve. In the mildest cases there may be no more than a little looseness of the bowels. In an ordinary case there will be considerable colic with violent purging for a day, and if the symptoms begin soon enough, there may be vomiting as well. In more serious cases, to these may be added some pyrexia, blood and mucus in the stools and, if the illness is prolonged, dehydration and prostration. In the absence of definite evidence that the condition is due to food poisoning the possibility of an acute appendicitis or abdominal influenza may arise. The differential diagnosis is discussed under the latter heading.

There are some individuals whose alimentary tracts are unduly irritable, wholly or in part, perhaps from a purely functional cause or perhaps they have had dysentery or ulcerative colitis. Such people have much more serious symptoms should they be poisoned or they may be upset by food which a normal person could take without harm.

Treatment.-In a case of food poisoning with really active vomiting and diarrhoea of several hours' duration, it is only slightly less foolish to use a purgative than a stomach pump. However half or one ounce of castor oil is usually given if the patient is seen within twelve hours regardless of how often the bowels may have acted. But if the diagnosis is certain and there has not been much diarrhoea, then it should be given, however long it may be before the case is seen. It is a good plan to follow the castor oil with paraffin for a few days owing to the tendency to subsequent -constipation. Frequently the doctor is called in when abdominal discomfort and diarrhoea continue for longer than the patient expects; then, of course, the 
symptoms are not due to the enduring presence of any of the offending food in the bowel, but to the irritation it has caused and the treatment is purely symptomatic (see below). The patient should always be allowed to quench his thirst, so long as he does not drink too quickly as this may cause a bowel action. In a really bad case the man is so ill that nothing given by the mouth or rectum is retained. When this happens small doses of morphia and atrophine must be given with the frequency that is needed to relieve the symptoms, to rest the patient and to allay his anxiety, and fluids will have to be supplied intravenously.

Diarrhoea in Chronic Alcoholism is due to gastro-intestinal catarrh and, sometimes, to achlorhydria. The patient is curiously boastful of his evacuative prowess, and dilates upon the benefits of frequent and loose actions; "grand stuff for the bowels," he says, making an excuse for his continued drinking. Although the gastritis is important the diarrhoea is not of much significance and he can certainly be allowed to keep it if this will help in the very difficult task of a cure. This is the only condition in which purgatives to the extent of two or three motions a day are justified and are indeed good treatment, for they rob the patient of one of his excuses for alcohol and they can be withdrawn later when he has improved. They are sometimes better given disguised as a " tonic," but this will depend on many things, the patient's intelligence for one.

\section{DIARRHOEA DUE TO PURGATIVES}

A friend of many years standing once came to me in great distress, complaining of colic, loss of weight and of not having had a bowel action for three months. Actually he was having six or eight slimy, watery evacuations and was taking "about three handfulls" of senna pods a day. Stupidity and ignorance of this degree are unbelievable until they are met. Here was a man of normal intelligence who mistook diarrhoea for constipation, thinking that unless the stool was formed there had been no bowel action. This is not an uncommon belief. There are others who recognise watery stools as diarrhoea and yet are content to let it continue, indeed they encourage it because it is only when the urge is really imperative that they can find time to visit the lavatory. Then there are those bowel-minded people who are constantly having feelings that " there is a little hold up" or "something wants to come away" and they go on dealing with the situation until something really does want to come away, and that something is the product of excessive irritation. There is nothing against a small daily dose of aperient to tide a man over some change of diet, environment or period of enforced inactivity, provided it is known that after a few days he can return to normal habits, but continued and, worse still, increasing doses are to be avoided somehow and usually it is not very difficult. The proper treatment of constipation is dealt with elsewhere in this number; here we have to consider the result of its improper treatment, usually by the patient himself.

The first, most important and most difficult part of the treatment of purgative addicts is to rid them of their prejudice. To the question " how often would your bowels be open without medicine" the invariable answer is " never," which only shows how prejudiced they are. Unless this attitude can be shaken they are incurable. The patient must have explained to him the impossibility of a proper, regular action without a formed stool and the irritant nature of purgatives, but the greatest care must be taken not to use the word " colitis," for this will only make him an even more enthusiastic bowel fan. Having, if possible, got the patient into a more malleable state of mind the treatment can begin. The dose of laxative is rapidly reduced so that none is used after the first week, and at the same time an ounce of paraffin a day is given, or whatever quantity is found necessary to be just noticeable at the anus, together with a teaspoonful of normacol t.d.s. These will supply lubrication for the bowel, and the semi-solid consistence to the stool which is needed for proper peristalsis. Every day after breakfast the patient goes to the lavatory and if the seat is too high, as it often is, he takes a footstool. If nothing has happened in fifteen minutes he comes out and does not return till the same time next day, unless he feels an urge, which must be obeyed at once. If there has been no action in three days a suppository is used, and, if that is not successful and the next morning is also fruitless, a small enema is given. Every time the bowels are open the regime begins again. More often than not neither suppositories nor enemas are required and if they are, they can soon be given up. If success is not met with after three weeks, then it is likely that the initial breaking down of prejudice has failed, and the patient is resisting rather than encouraging the natural call. Alternatively, he is a genuine case of some form of constipation, probably dyschezia. 


\section{ABDOMINAL INFLUENZA}

Often a new illness is trumpeted into an importance far beyond its deserts with a mass of articles and discussions, but no such fanfares greeted the appearance of abdominal influenza, indeed, it had a hard struggle for proper notice and it was not acknowledged by many consultants till years after its existence had been obvious to the humblest general practitioner. There were two reasons for this. Presented with the symptoms of a raised pulse and temperature, abdominal pain and vomiting, or diarrhoea, the doctor, if he wanted a second opinion, called in a surgeon who always diagnosed appendicitis, usually for the sake of safety, sometimes for other reasons; for a time it was thought that such symptoms were not due to the appendix and during that period the patients had their gall bladders out. Secondly, the milder cases gave no trouble to the doctor so they were not sent to hospital nor was there any consultation, thus consulting physicians either remained in true ignorance of the condition or would not admit its existence, not having seen it for themselves. Now abdominal influenza has been fully recognised for some years, yet it must be admitted that he would be a brave man, perhaps a foolish one, who would make a dogmatic diagnosis at his first visit in the absence of an epidemic. However, with careful watching and an appreciation of the points in the differential diagnosis there is no risk involved in an effort to save dozens of laparotomies. If there is any doubt and the evidence suggests that it would be safer to operate, then that is the proper treatment and it remains the proper treatment no matter what is the state of the appendix when it is removed.

The term abdominal influenza is used here instead of the more usual gastric influenza so as to embrace both the gastric and the intestinal types which occur in different epidemics. In some cases the symptoms are so severe that it must be assumed that both the stomach and the intestines are involved. .Here we are, of course, principally concerned with the intestinal type, which is the less common of the two. Sometimes the illness takes a day or two to develop. but much more often the onset is abrupt. Generally the first sign is that the patient feels cold and shivery without any obvious reasons, from then it may be only an hour before he has abdominal pain, violent purging and a little vomiting. (In the gastric type the two last symptoms will be reversed in severity.) The temperature is raised, IOI or more, and the pulse is up in proportion. The abdominal pain, which is usually central, and the purging, sometimes with blood, may continue for two or three days and then a gradual wane sets in or the symptoms abate as suddenly as they began, but the patient is left weak and, as in other types of influenza, convalescence may be slow. Such is the course of an ordinary case, but there is great variation in severity.

Diagnosis.-In distinguishing this disease from acute appendicitis one should note first the violence of the symptoms. It is rare to find such acute purging in appendicitis. In the first few hours of an intestinal influenza the patient is not so ill as he would be if he had an appendicitis giving the same pyrexia, pulse rate and diarrhoea. The abdominal pain and tenderness are more general in influenza and there is no definite rigidity, nor are there any localising signs on rectal examination. Other symptoms such as headache and sweating are characteristic of influenza, but not of appendicitis:- Finally, appendicitis is a lone disease and the other is epidemic, though too much importance must not be fastened to this. It is more difficult, as always, to make a diagnosis in mild cases. A really slight abdominal influenza cannot be distinguished from a mild appendicitis. Apart from their symptoms they can both appear well, but fortunately such patients do not demand an urgent diagnosis and they can be carefully watched for a rise of pulse rate or a suspicious localisation of the pain and tenderness.

There are two other conditions which have to be considered in the differential diagnosis -food poisoning and intestinal allergy. The history will usually clear the problem, if not, although both these disorders are sontetimes pyrexial, the temperature is lower in proportion to the symptoms than in abdominal influenza. Fortunately here the early differentiation is not so important as the treatment of food poisoning and intestinal influenza is much the same. If the case is one of intestinal allergy, even the most violent diarrhoea will subside rapidly, only to recur when the offending article of food is next taken.

Sometimes mild intestinal influenza is indistinguishable from the prodromal symptoms of catarrhal jaundice. After two or three days of trivial indisposition, a little temperature, nausea, some diarrhoea and slight abdominal pain, the symptoms subside, so the patient is told he 
has recovered and can go back to work. Next day he may be yellow in the conjunctivae, slightly so in the skin, urine dark, stools light, in fact very far from recovered. Though medically the sin is slight, it is catastrophic so far as concerns the patient's faith and the doctor's prestige.

The treatment of intestinal influenza is entirely symptomatic and is considered below.

\section{ULCERATIVE COLITIS AND OTHER ULCERATIONS OF THE BOWEL}

The term ulcerative colitis should really include all ulcerations of the colon, but it is only used to cover those cases in which the ulceration is not known to be caused by a specific organism or to cancer. Multiple ulceration anywhere in the colon is found in bacilliary and amoebic dysentery or ulcerative colitis, local ulceration in cancer and it stands to reason that, whatever the cause of the ulceration, it is likely to result in blood, pus and an excess of mucus in the stools. The other two common causes of intestinal ulceration, tuberculosis and typhoid, occasionally produce these symptoms, but never to such a striking degree as the lesions are much higher up in the bowel, being confined to the ileum and caecum in tubercle and to the small intestine in typhoid, so the blood, if any, is usually passed in altered form. Emphasis is placed on the nature of the stools because ulcerative colitis is the commonest cause of bloody diarrhoea in the country and because it is nearly always a striking feature of the illness by the time the doctor sees the patient. It is commonly supposed to attack introspective young adults; this is quite true, but it also attacks those of perfectly normal outlook. Sometimes the onset is acute, at others there may have been a history of intermittent looseness, for which the patient did not bother to seek advice, or if the motion was bloodstained he may have attributed it to piles.

On clinical grounds alone it is quite impossible to differentiate between ulcerative colitis and bacilliary dysentery as far as the bowel symptoms are concerned; nor is this surprising as the lesions themselves are indistinguishable. True, really violent symptoms early in the illness are most unusual in an ulcerative colitis, but in a Shiga or severe Flexner infection the victim may be quickly exhausted by innumerable imperative bowel actions, each one resulting only in a miserable pittance of blood and mucus passed with great tenesmus. Forty motions daily, of a sort, are not uncommon in such a case, twenty are rare in ulcerative colitis and so is tenesmus because the anal canal is not usually involved. Distant symptoms, such as headache and vomiting, are not often seen in ulcerative colitis. In short, extreme symptoms developing early make a diagnosis of dysentery probable. At the other end of the severity scale in dysentery come the Sonne infections, which account for some of the mild outbreaks of diarrhoea, such as are specially liable to occur in wartime. Such a case, if alone, might suggest mild ulcerative colitis.

Because of the difficulty of its early diagnosis, anyone with an unexplained temperature gets suspected of typhoid or paratyphoid, particularly if there is abdominal discomfort or diarrhoea. When the great day for the Widal comes, the diagnosis often has to be revised. Such a case might well be one of ulcerative colitis with the familiar insidious onset, but with less blood than usual. In adults the diarrhoea of tuberculous ulceration of the bowel is nearly always secondary to advanced phthisis, so the diagnosis will be self-evident.

Although no really striking differentiation in symptoms is possible, and not much help is to be had from examination of the patient, except perhaps his rectum, the actual diagnosis of ulcerative colitis or its many alternatives is usually a simple matter. The laboratory examination of the stools may show tubercle bacilli or entamaboe hystolitica or, by cultivation, one of the dysentery group; if so the question is settled with the single exception that tubercle bacilli can be found even when the bowel is healthy, having been swallowed and passed without doing any harm on the way.

Although these infective diseases are met with from time to time in this country, when blood and pus are found in the stools by far the most important differential diagnosis lies between ulcerative colitis and cancer of the bowel. Age is not much help, as cancer here is found in those of thirty or even less. The pelvic colon and rectum are the commonest sites and the diarrhoea often alternates with bouts of constipation. If a lump can be felt in the left iliac fossa it will have to be distinguished from diverticulitis or a faecal impaction, both of which may cause a slightly fluid stool with much mucus. In all cases a digital examination of the rectum is made. If this is negative and no diagnosis is forthcoming from the examination of the stools, 
then a sigmoidoscope should be used on every patient who passes blood and pus, or whose bowels change their habit for more than a week or two without apparent reason. The great majority of growths of the colon are within the reach of this instrument and ulcers can be seen in every case of ulcerative colitis. If it is passed to its fullest extent and no cause for the bleeding has been found, then it is almost certain that there is a growth beyond the range of the sigmoidoscope and this can be confirmed by $\mathrm{X}$-ray examination. The protoscope is not much help in making a diagnosis, for if nothing can be felt by the finger it is unlikely that its use, which is not pleasant for the patient, will make sigmoidoscopy unnecessary.

Only the course and treatment of ulcerative colitis can be considered here. The more recent the symptoms, the more likely and quicker the cure, sometimes three or four months are enough; but if the trouble is long standing at least a year is likely to be needed or a definite cure may be found to be impossible. As it is a long illness, for which no specific treatment is known, it is of the utmost importance to consider the victim's mental and general health. The doctor must realise that his own patience is going to be taxed, but that of his charge will be taxed much more, therefore he must warn the patient at the beginning that he will be lucky if there is any marked improvement in less than three months. This may sound abrupt, but it causes far less disappointment in the long run. He should also be told that his progress can only be judged by sigmoidoscopic examination and that the number or quality of the stools is no guide. This is not strictly true, but it saves the patient from becoming too interested in his bowel actions. His general health must be kept up with good food and plenty of it, only pips and skins being forbidden and those things which have a fibrous residue; vegetables should be given in purée form. After the temperature has been normal for about a week and if the frequency of the bowels permits, he can sit out of bed for a little, out of doors if possible. If he is not too ill, boredom and opportunity for introspection must be avoided by occupational therapy-in other words, he must be given something to do.

Bowel lavage is very helpful. Actually I do not think it much matters what fluid is used and I suspect water would be as good as anything; however, it is usual to add something to it, but solutions should not be too strong. Tannic acid $\frac{1}{2}-\mathrm{I}$ per cent, acriflavine $\mathrm{I}: 5,000$, potassium permanganate $I: 2,000$, and bicarbonate of soda I per cent are all used and in a long case it is encouraging for the patient to feel that a change is being made from time to time. The quantity depends on how much of the colon is involved, usually a pint or less is enough and it should be run in at gentle pressure twice a day directly after a bowel action. The patient should retain the fluid or, if this is impossible, after the actual irrigation has been returned a few ounces can be run in for retention. By mouth the diarrhoea can be treated symptomatically (see below), normacol, belladonna and some form of opium will probably be needed. When the stools begin to be formed the patient may have some slight abdominal discomfort due to the unfamiliar presence of anything but fluid in the bowel.

Mouth sepsis and sources of local irritation such as anal tags should be removed. Anaemia is so common as to be more a symptom than a complication and it must be treated by iron or blood transfusion.

As the case progresses, further sigmoidoscopies will have to be done, but no good purpose is likely to be served by this till after at least two months of treatment. If this examination is made comfortable for the patient, and there is no reason why it should not as pentothal can be given if necessary, then he will not dread its repetition. Sometimes one or two ulcers persist after the rest have healed and, but for them, the patient seems quite well. If he is discharged at this stage he is certain to relapse, so treatment must be continued until healing is complete. If possible a further examination should be made after he has been back at work for a month. The tendency to relapses is notorious in this disease, but their recurrence can be greatly reduced if these precautions are taken to ensure sound healing.

Sir Arthur Hurst, who has done so much work on ulcerative colitis, was the first to advise the trial of polyvalent anti-dysenteric serum. Occasionally this produces striking results in cases that do not improve with ordinary measures. When a patient is doing very badly one is sometimes tempted to consider surgery; the more the temptation can be resisted the better. But there are rare occasions when the doctor, despairing at his patient's cachexia from continued pouring of blood and pus from his bowel, is driven to advise surgery. Various operations have various advocates, but they all aim at a stoma through which the colon can be washed out. The results are poor, but it is only fair to say that the patient is usually in an emaciated condition 
by the time an operation is considered; yet it is hard to see why surgery should be advised more often, for it is almost as easy to wash out the whole colon from the anus upwards as from any kind of "ostomy" downwards. The one advantage gained from an ileostomy is that the colon is freed from faecès.

\section{DIARRHOEA DUE TO THE INDIVIDUAL}

Under this heading will be considered those cases in which diarrhoea is caused through some inherent fault of the individual.

(a) Gastro-Intestinal Allergy.-In this condition there is nothing wrong with the food itself except in so far as the particular individual is concerned; to others it is good, to him it is poison. The usual manifestations are abdominal pain and diarrhoea. It is odd that this cause of diarrhoea is not considered more often. While everyone recognises that such symptoms may occur after, say, strawberries or lobster, the fact that they may happen after milk or mutton is commonly overlooked. The family history and other manifestations of allergy in the patient will help towards a diagnosis, but even without these pointers it should be considered in all cases of unexplained recurrent diarrhoea. Unfortunately skin tests are no proof one way or another as the extracts used are made from raw foods, which may be changed by cooking. This difficulty might be overcome, but not the chance of the allergy being due to the products of digestion rather than to the original food. The only way to arrive at the source of the trouble is by elimination. During a test period the patient avoids all complicated dishes such as stews, hashes, salads and sauces. He keeps a diary of everything he eats and the resulting symptoms, if any. Then, for a while, he bases his diet on those foods he has taken on symptom-free days and to them he gradually adds the articles taken on the other days. In this way the offending foods can be traced with certainty. It might be objected that this process would tend to food consciousness and introspection, but it would not be applied until the diagnosis of gastrointestinal allergy had been made and, if that were correct, no amount of extroversion or of fighting against the symptoms would be of any avail.

It is important to remember that allergic manifestations may develop at any period in life and may change, so if a patient's symptoms recur he may need reassessing. If further foods are found to be causing trouble a fresh try-out of the original offenders should be made so that the patient does not have more restrictions in his diet than necessary.

The differential diagnosis has been considered under the heading of abdominal influenza.

(b) Gastrogenous Diarrhoea is consequent upon hypochlorhydria or achlorhydria. In these conditions swallowed bacteria, which would have some difficulty in surviving a normally acid gastric juice, can reach the bowel unharmed; thus the chance of all kinds of intestinal infection, such as dysentery or typhoid, are increased. With this easy passage of bacteria through the stomach, diarrhoea can be caused by an infection of the upper respiratory tract. This is sometimes seen in recurring sinusitis and of course many people have it each time they get a head cold.

Apart from these swallowed infections there are other possible causes of gastrogenous diarrhoea. With deficient secretion gastric digestion, especially of meat and vegetables, is poor and the food is improperly liquified, partly because of its rapid passage through the stomach and partly through a shortage of acid and pepsin in the gastric juice, so when it reaches the bowel it causes irritation. Furthermore, the lack of acid leaving the pylorus allows of a great increase of bacteria in the intestine. Diarrhoea is common enough in pernicious anaemia and in chronic alcoholism, but it is by no means a necessary complication of achlorhydria, which more often than not is symptomless. When diarrhoea occurs after gastro-enterostomy it is, of course, because of the too rapid passage of food from the mouth to the intestine.

On symptoms and history the diagnosis of gastrogenous diarrhoea is not often possible unless it is associated with some swallowed infection or with a known achlorhydria. However stools which are foul smelling, slimy, alkaline and which contain a great excess of meat fibres make the diagnosis a probability. A test meal is usually unnecessary for confirmation, as this can be obtained more easily by the success of the treatment. From 20 minims to I drachm of hydrothloric acid in a lemon drink should be given with each meal with or without pepsin. If the diagnosis is correct the symptoms will soon disappear. The treatment can sometimes 
be diminished or discontinued after a few weeks or replaced by the more convenient acidolpepsin tablets:

(c) Intestinal Carbohydrate Dyspepsia.-In this condition there is an inadequate supply of starch-splitting ferment in the pancreatic juice so that starch reaches the colon instead of being completely digested in the upper part of the small intestine. The result is a tremendous formation of gas, with great discomfort, some colic, much flatus and occasional diarrhoea. The diagnosis is not difficult, flatulence being the important symptom, and confirmation can be had by the finding of a big excess of starch in the stools. Treatment is largely diatetic, vegetable starch, which abounds in all roots, and rice being forbidden and bread restricted. It is almost impossible, especially in wartime, to make the diet entirely starch free, but takadiastase, gr. I or 2 , with each meal will get over this difficulty.

In some people a digestive hurry all the way from the mouth to the large intestine occurs and this may result in a combination of the two foregoing conditions, Gastrogenous Diarrhoea and Intestinal Carbohydrate Dyspepsia. In such patients both meat fibres and starch will be in excess in the stools and they should receive both the appropriate treatments.

(d) Nervous Diarrhoea, in its true form is not nearly so common as is generally supposed. The two emotions that are said most to favour it are fear and excitement, but it is doubtful if either of them cause diarrhoea in those who are otherwise perfectly healthy. War provides plenty of circumstances in which these two feelings have full play. Going into battle or on patrol, knowing that survival is less likely than death and that unwounded survival is less likely still, must be just about as exciting and frightening an experience as this world has to offer. In the last war a close association with this kind of life brought to my notice not one single instance of diarrhoea, yet I often heard men remarking it as a peculiar fact that there was never any desire for last moment defaecation, although frequency of micturition was so common as to be almost inevitable (though they did not use these actual words). I have asked various friends who are Medical Officers in the Royal Air Force if they have ever come across diarrhoea among their pilots or crews, but none of them have ever met it and these Medical Officers are in such close contact with their charges that they would be sure to hear about it if the symptoms occurred with any frequency. The actual sight of impending death in the form of a poised bayonet or burning fusilage may cause a bowel evacuation, but this is of no clinical interest.

The diagnosis of nervous diarrhoea must be made with great deliberation and the question of any contributing factors carefully investigated. The nervous after-dinner speaker may have had a temporary hypochlorhydria and so his diarrhoea will be gastrogenic, that of the anxious man may be aggravated by hyperthyroidism. Such and other possibilities must be considered, for it is no good saying that the diarrhoea is due to the patient being highly strung or introspective and just dismissing it as of no account, for sooner or later the desire to defaecate may come on whenever he is not near a lavatory and be a great drag on his life.

The treatment will be that of any associate cause that may be found and if there are none then Luminal may be of help and failing anything else slight constipation must be induced and re-education attempted.

\section{THE SYMPTOMATIC TREATMENT OF DIARRHOEA}

The commonest cause of diarrhoea is diarrhoea itself. The presence of liquid in the bowel further down than is normal, that is anywhere beyond the caecum, acts as an irritant, also the very fact that it is liquid means that it has made its journey with undue speed and so probably contains products of imperfect digestion, which are also irritant. So whatever the primary cause of diarrhoea may be, it is very easy for a vicious circle to become established unless the symptom is treated as well as the cause; indeed, as we have already seen, there is generally not much else that can be done.

The symptom of diarrhoea can be attacked in three ways, by lessening the fluid consistence of the stool, by decreasing peristalsis, and by the use of astringents. Often a combination of methods is advisable. If the stool is made more solid it will not be able to run through the colon as if it were a sewer, it will depend upon peristalsis for its journey, and peristalsis will not be so active as the faecal mass will be less irritating. Both these reasons will help its passage to take longer. Psyllium seeds, normacol, coreine and isogel are all identical in their action; 
in the bowel they absorb moisture and form a gelatinous mass. They must always be taken dry and can be used in any quantity desired. Perhaps it is because they are so often prescribed for chronic constipation that they tend to be forgotten in diarrhoea. They can be given alone in mild cases, in more severe ones they form a most useful addition to other treatment.

Peristalsis can be directly decreased by opium, and this is by far the most effective way of treating diarrhoea. Morphia addicts not only harm themselves; they do great damage by inducing quite an unwarranted timidity in the use of opium and all its derivatives. Tinct. opii, pulv. cret. aromat. cum opio and pil. codeinae are the most useful preparations. Not only can they be used in acute attacks, but they can and should be given in long cases of ulcerative colitis to give the patient necessary rest from the bedpan. If vomiting is present morphia may be used by injection, but not otherwise as it is not so effective in checking diarrhoea as opium. Unfortunately morphia sometimes causes vomiting. There are occasions when this risk must be taken, but it is as well to begin with a small dose.

In the astringent group the subnitrate and the carbonate of bismuth stand alone. They are far more widely used than anything else for diarrhoea, and can be given in combination with opium; while mist. bismuthi et sodae is probably the most commonly prescribed mixture there is and for mild diarrhoea it is excellent. The only other astringent worth mentioning is tannic acid, which is sometimes given by the mouth, but more often as a $\mathrm{I}$ per cent solution for lavage.

Colic and flatulence often go arm-in-arm with diarrhoea. Colic can frequently be reduced by atrophine, belladonna or tincture of hyosciamus, but wind is the bugbear of all mankind especially when it is in the bowel. Charcoal or kaolin sometimes help; if they do; the patient is lucky.

\title{
Clinical Page
}

\section{ANOTHER RARE TUMOUR OF ILEOCAECAL ORIGIN}

\author{
By M. D. SHEPPARD, M.B., F.R.C.S.(ENG.)
}

A woman of forty-nine first fell ill with generalised abdominal pains five years ago, accompanied by vomiting. It was diagnosed as gastritis and she improved with treatment, only to suffer a relapse a fortnight later, when she vomited blood. Ever since then, she has had attacks of acute abdominal pain every four to five weeks, but she has not been sick. During these years she quite often suffered from diarrhoea, being at times completely prostrated by the frequent bowel actions.

The pain was described as a general, abdominal colicky pain. At the height of the spasms of pain a lump would appear in the right iliac fossa. This was followed by a loud gurgling noise and the disappearance of the pains.

She has a family of three, and her periods stopped quite uneventfully four years ago.

Examination showed her to be an intelligent woman, very thin, rather pallid and grey, and thoroughly resigned to her complaint. I was fortunate enough to witness the manifestation she had described. She warned me that they were due, and after a few severe colicky pains, a rounded smooth tumour appeared in the right iliac fossa, which felt quite firm. It was obviously a powerful peristaltic wave in a loop of grossly hypertrophied intestine, for it disappeared completely, leaving no more than the sensation of an ill-defined mass in the right iliac fossa. This was followed by loud borborigmi.

Vaginal and rectal examination suggested an indefinite mass high up in the right side of the pelvis, but no more

She was having two or three loose bowel actions daily, but sigmoidoscopy revealed a normal mucuous membrane and no cause for diarrhoea. A barium enema flowed uninterruptedly as far as the caecum, but could not be forced into the ileum. A barium follow-through showed some delay in the terminal ileum, and dilated small gut, but no evidence of the string sign. Blood count, pulse, temperature and urine were all normal.

In view of the long history, diarrhoea, and obstructive signs and symptoms, a diagnosis of Crohn's disease was made, and under gas, oxygen and ether anaesthesia, the abdomen was ex- 